\title{
Sprint interval training versus moderate-intensity continuous training during inpatient rehabilitation after spinal cord injury: a randomized trial
}

\author{
Jonathan C. Mcleod ${ }^{1} \cdot$ Herrington Diana ${ }^{2} \cdot$ Audrey L. Hicks $^{1}$
}

Received: 21 May 2019 / Revised: 7 August 2019 / Accepted: 9 August 2019 / Published online: 28 August 2019

(c) The Author(s), under exclusive licence to International Spinal Cord Society 2019

\begin{abstract}
Study design Randomized trial.

Objectives To evaluate the effectiveness of a 5-week sprint interval training (SIT) protocol on an arm-crank ergometer in individuals with sub-acute spinal cord injury (SCI).

Setting Inpatient rehabilitation.

Methods Individuals with SCI $(N=20 ; 9$ tetraplegia/11 paraplegia; time since injury, $14-182$ days; age, $46 \pm 16$ years; $15 \mathrm{M} / 5 \mathrm{~F}$ ) were randomized to SIT or moderate-intensity continuous training (MICT). SIT consisted of $3 \times 20 \mathrm{~s}$. 'all-out' cycle sprints $(\geq 100 \%$ peak power output) interspersed with $2 \mathrm{~min}$ of active recovery ( $10 \%$ peak power output; total time commitment, 10 mins). MICT involved 20 min of cycling (45\% peak power output; total time commitment, 25 mins). Both training interventions were delivered 3 times/week for 5 weeks. Heart rate and Borg's Rating of Perceived Exertion (RPE; 6-20) were monitored throughout training sessions. Maximal and sub-maximal power outputs were assessed on an armcrank ergometer. Exercise enjoyment, exercise self-efficacy, and pain were assessed at the end of the intervention.

Results During training sessions, heart rate (135 bpm vs. $119 \mathrm{bpm} ; p=0.05$ ), peripheral RPE (16 vs. $12 ; p=0.000$ ), and central RPE (15 vs. $11 ; p=0.004)$ responses were higher in the SIT group, yet total work performed was greater in MICT. Peak power output increased significantly with training (36\%), with no difference between groups (39\% vs. 33\%; $p=$ 0.524). Similarly, improvements in sub-maximal power output were not different across groups. There were no betweengroup differences in exercise enjoyment $(p=0.385)$, exercise self-efficacy $(p=0.930)$, or pain $(p=0780)$.

Conclusions Five weeks of SIT improved physical capacity to the same extent as MICT in individuals with sub-acute SCI, despite a significantly lower time commitment with SIT.
\end{abstract}

\section{Introduction}

Early after the stabilization of a spinal cord injury (SCI), and managing the associated acute, secondary conditions, patients are admitted to inpatient rehabilitation, where preparation for a return to the community or independent living environment become the main goals [1]. The benefits of regular exercise (aerobic training and resistance training) on

Audrey L. Hicks

hicksal@mcmaster.ca

1 Department of Kinesiology, McMaster University, Hamilton, ON, Canada

2 Regional Rehabilitation Centre, Hamilton Health Sciences, Hamilton, ON, Canada alleviating numerous health complications associated with SCI, augmenting functional recovery, and preventing cardiovascular deconditioning following injury are well established $[2,3]$. Thus, it is no surprise that physical therapists prescribe exercise during SCI inpatient rehabilitation.

Persons with SCI typically utilize an arm-crank ergometer to perform aerobic exercise training [3-10]. SCI-specific physical activity guidelines recommend that for cardiorespiratory and health benefits, community-dwelling individuals with SCI should accrue, at minimum, three bouts of $20 \mathrm{~min}$ of moderate to vigorous-intensity activity per week [11]. Unfortunately, most individuals with SCI undergoing inpatient rehabilitation spend very little time at the moderate to vigorous intensities required for enhancing health benefits and improving ones physical capacity- this represents a lost opportunity to maximize rehabilitation [12]. Furthermore, decreased lengths of stay during inpatient rehabilitation in 
North America ( $\approx 40$ days) translates to less time to optimize neurological, cardiovascular, and musculoskeletal health prior to discharge [13, 14]. Clearly, the utilization of time efficient, yet effective, exercise regimes during SCI inpatient rehabilitation is warranted.

Recently, low-volume high intensity interval training has garnered a lot of attention for improving cardiometabolic health in non-disabled individuals $[15,16]$. Briefly, interval training consists of brief bursts of vigorous-intensity activity, interspersed with periods of low-active recovery [15]. Sprint interval training (SIT) represents a type of interval training involving "all-out" efforts, at intensities corresponding to $\geq 100 \%$ of one's peak power output $[15,16]$. Gillen et al. demonstrated that 12 weeks of SIT on a leg ergometer in inactive men improved cardiorespiratory fitness to the same extent as moderate-intensity continuous training (MICT), despite a five-fold lower exercise volume and training time commitment [16]. For individuals with SCI, lack of time is a commonly cited barrier for engaging in physical activity, and thus, the minimal training duration for SIT may be desirable for many individuals [17].

A potential advantage to employing SIT in persons with SCI is that it enables them to perform bouts of vigorousintensity exercise [18]. Astorino and Thum demonstrated that one session of SIT elicited higher metabolic, cardiovascular, and cardiorespiratory strain than MICT in individuals with SCI [19]. To our knowledge, there has been only one study evaluating a SIT protocol on an arm-crank ergometer in community-dwelling individuals with SCI, where improvements in peak power output were seen after the 2week training program [20]. However, there are currently no published trials comparing the effects of SIT to MICT in individuals with SCI undergoing inpatient rehabilitation.

The purpose of this study was to evaluate the effectiveness of a 5-week, thrice-weekly $10 \mathrm{~min}$ SIT program, and compare outcome measures to a traditional $25 \mathrm{~min}$ MICT program on an arm-crank ergometer in individuals with SCI undergoing inpatient rehabilitation. The primary objective of this study was to compare improvements in peak power and sub-maximal power output across both MICT and SIT. Secondary objectives of this study included comparing (1) exercise responses (i.e., heart rate and perceived exertion), (2) exercise enjoyment, (3) exercise self-efficacy, and (4) pain perceptions across both MICT and SIT. As this was an exploratory study, we were also interested in determining if lesion level (i.e., tetraplegia vs. paraplegia) affected any of the aforementioned outcomes. It was hypothesized that 5 weeks of SIT and MICT would induce similar changes in maximal and sub-maximal power output, exercise selfefficacy, and exercise enjoyment, despite large differences in training volume and time commitment. It was also hypothesized that SIT would elicit higher levels of cardiovascular strain than MICT but would be well tolerated.

\section{Methods}

\section{Participants}

Individuals with SCI were recruited as a convenience sample from an inpatient SCI rehabilitation clinic (Regional Rehabilitation Centre, Hamilton Health Sciences, Hamilton, Ontario, Canada). Recruitment began in June 2017 and was terminated in June 2018; this trial was registered retrospectively under Clinicaltrials.gov identifier NCT03709095. Participants eligible for the study were between the ages of 18 and 65 , with a SCI of less than 365 days, and injuries below the second cervical vertebrae. We excluded individuals with type II diabetes, cardiovascular disease, cancer, were re-admitted to inpatient rehabilitation following discharge, pre-existing shoulder injuries, or other medical comorbidities precluding safe participation in arm-ergometry training. The Hamilton Integrated Research Ethics Board approved all study procedures. All participants gave written informed consent. We did not plan for an intention to treat analysis, but instead a treatment exposure analysis, where only participants that complied with the intervention were included in the final analyses.

\section{Experimental design}

All training sessions and performance tests were completed on the Monark 881E Rehab Trainer (Patterson Medical Supply, Ontario, Canada) in the physical therapy gym, at the Regional Rehabilitation Centre. Care was taken to ensure that an arm-crank ergometer was positioned such that the axis of the crank arm was horizontally aligned with the participant's shoulder and the arms were slightly flexed at the furthest point of reach [2]. After baseline testing, using a random number computer generator (https://www. graphpad.com/quickcalcs/randomize1/) and concealment of allocation, J.C.M randomized (1:1 allocation ratio) participants to perform MICT or SIT, three times a week, for 5 weeks. As we recruited from a convenience sample, we were unable to utilize any stratification or minimization techniques. Peak power output, sub-maximal exercise performance and exercise self-efficacy were assessed at baseline (pre-training) and $72 \mathrm{~h}$ following the final training session (post-training). Exercise enjoyment and pain perceptions were assessed at the post-training time point only.

\section{Inpatient rehabilitation: physical therapy}

During the conduct of the study, participants were performing daily physical therapy. Activities performed during physical therapy sessions ranged between participants, but for the most part, consisted of: bed mobility activities (i.e., rolling, scooting, re-positioning), pre-gait activities (i.e., 
parallel bars, standing frame), range of motion/stretching activities (i.e., active/passive range of motion, manual/ orthopaedic treatment), upper-body strength training (i.e., pulleys, dumbbells, elastic bands, gravity eliminated sling), and aerobic training (i.e., leg ergometer with functional electrical stimulation, recumbent stepper). As the physical therapy sessions were tailored to meet the unique goal(s) of the participants at discharge, we did not standardize physical therapy sessions across groups. However, the order in which participant's performed their training sessions and exercise performance tests relative to performing their physical therapy session was maintained throughout the entirety of the study (i.e., participants were given the option to perform arm-crank ergometry only before or only after physical therapy). Participants were asked to refrain from using an arm-crank ergometer outside of the study; however, performing aerobic exercise using other modalities were acceptable (e.g., recumbent stepper).

\section{Assessment of peak power output}

Participants underwent a maximal graded exercise test on an arm-crank ergometer to assess peak power output at pretraining and post-training. After a 1 min warm-up with no resistance, power output was increased in a ramp-like matter by 10 watts/min for persons with paraplegia, and 5 watts/ min for persons with tetraplegia [21]. Participants were asked to maintain a constant, self-selected cadence between 60-80 revolutions per minute. Participants continued to pedal until one of the following criteria were met: (i) volitional exhaustion or (ii) they were unable to maintain their self-selected pedaling cadence for 20 consecutive seconds [21]. Peak power output (in watts) was the outcome of interest for this test and was defined as the highest workload participants could maintain for at least $30 \mathrm{~s}$. Heart rate (Polar T31, Quebec, Canada) was measured continuously, and the highest value averaged over the final $30 \mathrm{~s}$ of the test was taken as the heart-rate peak.

\section{Assessment of submaximal arm-crank ergometry performance}

The Discontinuous University of Toronto Arm Crank Protocol $[6,22]$ was used to assess power outputs at three submaximal workloads on an arm-crank ergometer at pretraining and post-training. Participants performed three 5min steady state workloads on an arm-crank ergometer at power outputs corresponding to central (heart and lungs) and peripheral (arms) Borg's Ratings of Perceived Exertion (RPE; 6-20 categorical scale [23]) of 8, 10, and 12 [6]. Participants were given a minimum of 2 min, and a maximum of $5 \mathrm{~min}$ of rest in between each workload. Submaximal power output (in watts) at each workload was the outcome of interest for this test. For each workload, heart rate was averaged over the final $2 \mathrm{~min}$ and expressed as a percentage of heart-rate peak achieved during the peak power output test at pre-training-this was defined as relative heart rate.

\section{Training intervention}

Exercise sessions were offered to participants three times per week, for 5 weeks. Adherence to the exercise intervention was expressed as a percentage of the number of sessions completed over the maximum number of sessions that could be completed. All exercise sessions began with a 2-min warm-up and concluded with a 3-min cool-down $(\approx 10 \%$ of peak power output). The SIT protocol consisted of $3 \times 20 \mathrm{~s}$ "all-out" efforts to elicit a peripheral RPE of 16 ( $\geq 100 \%$ of peak power output achieved at pre-training), at a self-selected cadence $(\approx 100$ revolutions per minute for persons with paraplegia, and $\approx 85$ revolutions per minute for persons with tetraplegia [16]). Each sprint was separated by $120 \mathrm{~s}$ of active recovery ( $\approx 10 \%$ of peak power output). Participants randomized to MICT performed $20 \mathrm{~min}$ of armergometry at a self-selected cadence at an intensity corresponding to a peripheral RPE of 12 (45\% of peak power output $[19,24])$. Total training duration for each session (including warm-up and cool-down) was $10 \mathrm{~min}$ for SIT and $25 \mathrm{~min}$ for MICT. Heart rate was recorded continuously and averaged for an entire training session (excluding warm-up and cool-down). Peripheral and central RPE were measured at the end of each sprint (SIT group) or at 5, 10, 15 , and $20 \mathrm{~min}$ of exercise (MICT group) and were averaged together for an entire training session. In order to accommodate training adaptation, the wattage on the armcrank ergometer was increased to maintain a peripheral RPE of 16 for SIT, and 12 for MICT. Exercise workload was expressed as a percentage of power output over the peak power output achieved at pre-training. Exercise volume (expressed in kilojoules $[\mathrm{kJ}]$ ) was calculated as the product of mean power and total duration of each session.

\section{Assessment of exercise satisfaction, exercise self- efficacy and pain}

After completion of the final training session, participants completed The Physical Activity Enjoyment Scale to assess their level of enjoyment with the training intervention they received. This questionnaire has 18 bi-polar questions (e.g., I enjoy it, I hate it) scored using a 1-7 likert scale. Answers to each question were summed together in order to acquire a cumulative score that can range from 18 (least enjoyment) to 126 (most enjoyment). The Physical Activity Enjoyment Scale is a valid and reliable measure that has been previously used in persons with SCI [24, 25]. 
Exercise self-efficacy was measured using the SCI Exercise Self Efficacy Scale [26]. The SCI Exercise Self Efficacy Scale consists of 10 questions and instructs respondents to indicate on a four-point likert scale $(1=$ not true, $4=$ always true) how confident they are with performing and carrying out regular physical activities and exercises. Answers to each question were summed together to acquire a cumulative score that can range from 10 (low self-efficacy) to 40 (high self-efficacy). This questionnaire contains high internal consistency (chronbach's $\alpha=0.926$ ) [26].

Pain perceptions were measured using the questionnaire created by Pelletier et al. [27]. Using a 7-point scale, participants rated how much shoulder pain, bodily pain, and physical discomfort they typically experience throughout the day and how much they experienced during arm-crank ergometry training $(1=$ none, $7=$ extreme pain). Answers to each question were summed together to acquire a cumulative score that can range from 3 (no pain) to 21 (extreme pain).

\section{Statistics}

The Kolmogorov-Smirnov Test and Levene's Test of Equality was used to assess normality and homogeneity of variance. All dependent measures were analyzed using a three-way mixed ANOVA (group [MICT, SIT] $\times$ lesion level [tetraplegia, paraplegia] $\times$ time [pre-training, post-training]). Differences in change scores were analyzed using a two-way between groups ANOVA (group [MICT, SIT] $\times$ lesion level [tetraplegia, paraplegia]). Tukey's posthoc test was used where appropriate. Statistical analyses were performed using IBM SPSS Statistics, version 20.0. Significance was set at an alpha level $<0.05$. Values are presented as mean $(95 \%$ confidence interval [CI]: lower bound, upper bound).

\section{Results}

\section{Baseline characteristics, program adherence, and adverse events}

Twenty participants completed the intervention (MICT $n=$ 10, SIT $n=10$; Fig. 1). Participant characteristics are displayed in Table 1. The attendance rate was $89 \%$ for MICT and $86 \%$ for SIT. One adverse event occurred during a SIT session (post-exercise hypotension [session \#1; injury level, T10; ASIA, C]).

\section{Exercise data, and heart rate, and ratings of perceived exertion responses to training}

Exercise workload during the SIT and MICT corresponded to $154 \%$, and $64 \%$ of peak power output achieved at
Fig. 1 Consolidated Standards of Reporting Trials (CONSORT) flow diagram

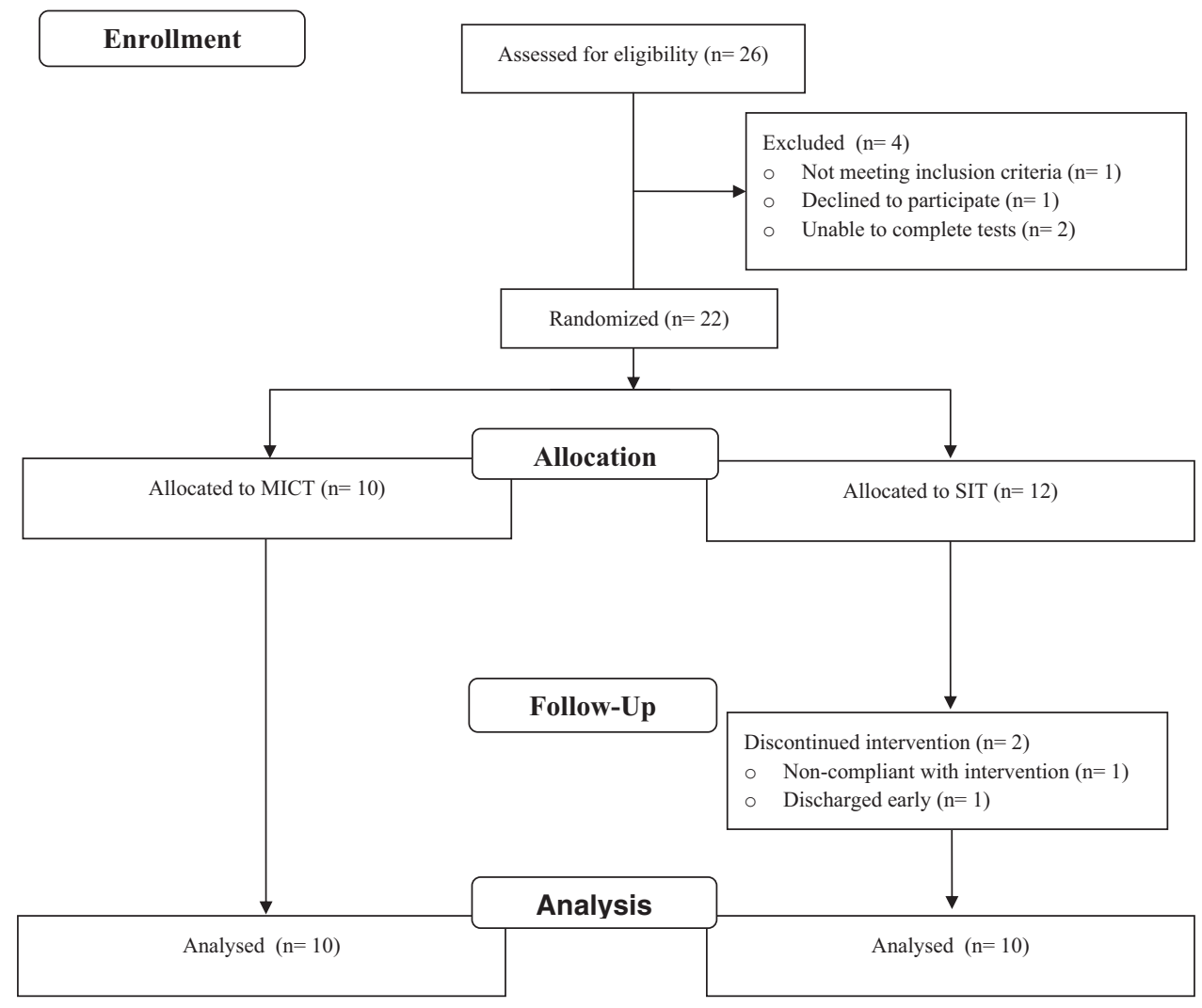


pre-training, respectively. Over the course of the 5 weeks of training, the average volume performed during SIT was lower than MICT (Table 2).

Six participants were using heart-rate lowering medication (Table 1) and were excluded from the heart-rate analysis (sample size used: $n=14$, MICT $n=7$, SIT $n=7$ ). Compared to the MICT group, mean heart rate, central RPE, and peripheral RPE were higher for the SIT group (Table 2). There was a trend for a group $\times$ lesion level interaction for mean heart rate (Fig. 2a; $p=0.065$ ), such that the mean heart rate for persons with tetraplegia appeared to be no different between SIT and MICT (mean group difference: 1 bpm, 95\% CI: -31, 33), whereas for persons with paraplegia, mean heart rate appeared to be higher for SIT than MICT (mean group difference: 39 bpm, 95\% CI: 5, 73). Conversely, there was no group $\times$ injury level interaction for central RPE (tetraplegia mean group difference: 3, 95\% $\mathrm{CI}:-1,7$; paraplegia mean group difference: $4,95 \% \mathrm{CI}$ : 1, 7; $\mathrm{P}=0.565$; Fig. 2b) or peripheral RPE (tetraplegia

Table 1 Participant characteristics

\begin{tabular}{lll}
\hline Parameter & MICT $(n=10)$ & SIT $(n=10)$ \\
\hline Age, years & $45 \pm 17$ & $47 \pm 15$ \\
Sex & & \\
$\quad$ Male & $5(50 \%)$ & $10(100 \%)$ \\
$\quad$ Female & $5(50 \%)$ & $0(0 \%)$ \\
TSI, days & $56 \pm 42$ & $72 \pm 68$ \\
Lesion level & & \\
$\quad$ Tetraplegia & $5(\mathrm{C} 2-\mathrm{C} 7)$ & $4(\mathrm{C} 2-\mathrm{C} 4)$ \\
$\quad$ Paraplegia & $5(\mathrm{~T} 8-\mathrm{L} 4)$ & $6(\mathrm{~T} 7-\mathrm{L} 2)$ \\
ASIA class & & $1(10 \%)$ \\
$\quad$ A & $1(10 \%)$ & $0(0 \%)$ \\
$\quad$ B & $0(0 \%)$ & $4(40 \%)$ \\
C & $4(40 \%)$ & $5(50 \%)$ \\
$\quad$ D & $5(50 \%)$ & $3(30 \%)$ \\
Heart-rate lowering medication ${ }^{\mathrm{a}}$ & $3(30 \%)$ &
\end{tabular}

TSI time since injury, ASIA American Spinal Injury Association ${ }^{\mathrm{a}} \beta$-blockers or $\mathrm{Ca}^{2+}$ channel blockers mean group difference: $3,95 \% \mathrm{CI}: 1,5$; paraplegia mean group difference: 4, 95\% CI: 2, 6; $p=0.428$; Fig. 2c).

\section{Peak power output test and sub-maximal exercise performance test}

Peak power output and sub-maximal exercise performance increased after training (Fig. 3). Peak power output increased by $39 \%(95 \%$ CI: 18,60$)$ for SIT, and 33\% $(95 \%$ CI: 15,50$)$ for MICT, with no difference between groups (mean group difference: $6 \% ; 95 \% \mathrm{CI}:-19,31 ; p=0.524$; Fig. 3b). Improvements in peak power output were no different across persons with paraplegia or tetraplegia (Fig. 3b). Similarly, there were no between-group differences in improvements in power output during sub-maximal workloads I (mean group difference: $-4 \%$; $95 \% \mathrm{CI}:-15$, 23; $p=0.831$ ) II (mean group difference: 2\%; $95 \%$ CI: $-18,22 ; p=0.720$ ), and III (mean group difference: 0\%; 95\% CI: $-22,22 ; p=0.800$; Fig. 4).

\section{Exercise satisfaction, exercise self-efficacy, and perceived pain responses}

Results of the questionnaires are presented in Table 3. There were no group differences in exercise enjoyment, changes in exercise self-efficacy, and pain.

\section{Discussion}

The novel finding of the present investigation was that 5 weeks of SIT improved peak power output, and submaximal arm-crank ergometry performance to the same extent as MICT in individuals with SCI undergoing inpatient rehabilitation. The SIT protocol involved a total of 1 min of intense intermittent exercise, with a time commitment of 10 min per session, whereas MICT consisted of $20 \mathrm{~min}$ of continuous cycling with a $25 \mathrm{~min}$ total time commitment. The present work also demonstrated that SIT exerted a higher cardiovascular response and perceived

Table 2 MICT vs. SIT training session responses

\begin{tabular}{|c|c|c|c|c|}
\hline & \multirow[t]{2}{*}{ MICT } & \multirow[t]{2}{*}{ SIT } & \multicolumn{2}{|l|}{ ANOVA } \\
\hline & & & $\Delta$ between groups & Group $p$-value \\
\hline $\begin{array}{l}\text { Exercise workload, \% peak } \\
\text { power output }\end{array}$ & 64 (17, 95\% CI: 52, 77) & 154 (55, 95\% CI: 114,193$)$ & 90 (41, 95\% CI: 51,128$)$ & 0.000 \\
\hline Exercise Volume, kJ & 37 (16, 95\% CI: 25, 49) & 13 (8, 95\% CI: 7, 18) & $-24(13,95 \%$ CI: $-12,-37)$ & 0.000 \\
\hline Heart rate, bpm & $119(16,95 \%$ CI: 104,135$)$ & $135(29,95 \%$ CI: 108, 163) & $15(24,95 \%$ CI: $-13,43)$ & 0.05 \\
\hline Central RPE, 6-20 & $11(3,95 \%$ CI: 9,13$)$ & $15(2,95 \%$ CI: 14,16$)$ & $4(2,95 \%$ CI: 2,6$)$ & 0.004 \\
\hline Peripheral RPE, 6-20 & $12(1,95 \%$ CI: 11,13$)$ & $16(2,95 \%$ CI: 15,17$)$ & $4(2,95 \%$ CI: 2,6$)$ & 0.000 \\
\hline
\end{tabular}

Values represent mean responses across all training sessions (standard deviation, 95\% CI: lower bound, upper bound) 

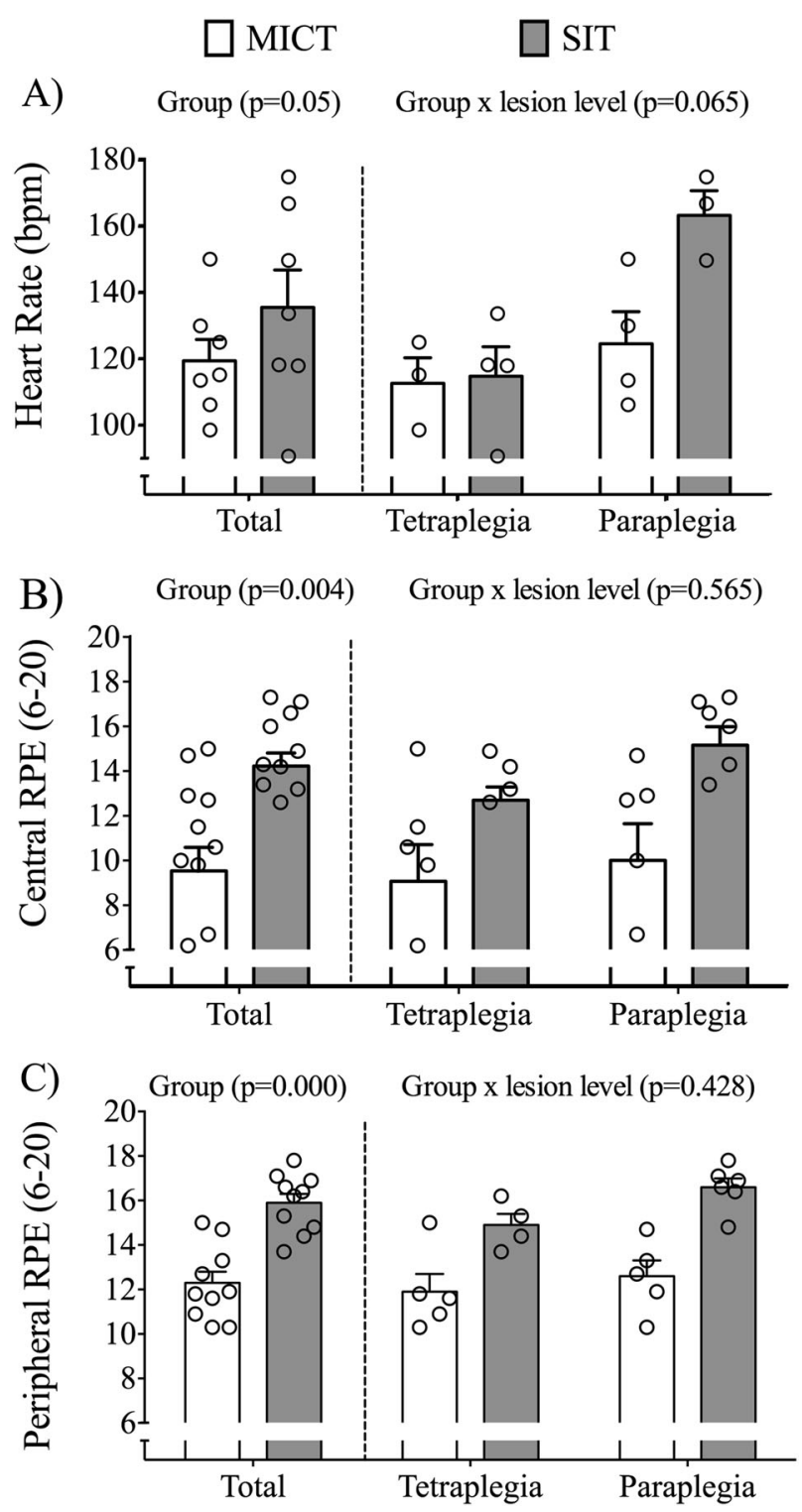

Fig. 2 Mean and individual data for heart rate (a), central RPE (b), and peripheral RPE (c) responses during the 5-week training intervention. Mean \pm SEM are shown

exertion than MICT, yet SIT was equally enjoyable as MICT and well tolerated. Out of all of the SIT sessions conducted, only one adverse event (post-exercise hypotension) occurred. Compared to able-bodied individuals, postexercise hypotension is more likely to occur in persons with SCI during a bout of arm-crank ergometry, and this can be attributed to decreased vasomotor control below the level of the lesion [2]. Although the current study demonstrated a low rate of adverse events with vigorous-intensity exercise on an arm-crank ergometer, clinical practitioners are encouraged to monitor blood pressure for recently injured individuals while implementing SIT.

Another important finding of the study was that, during training sessions, individuals performing SIT had a higher
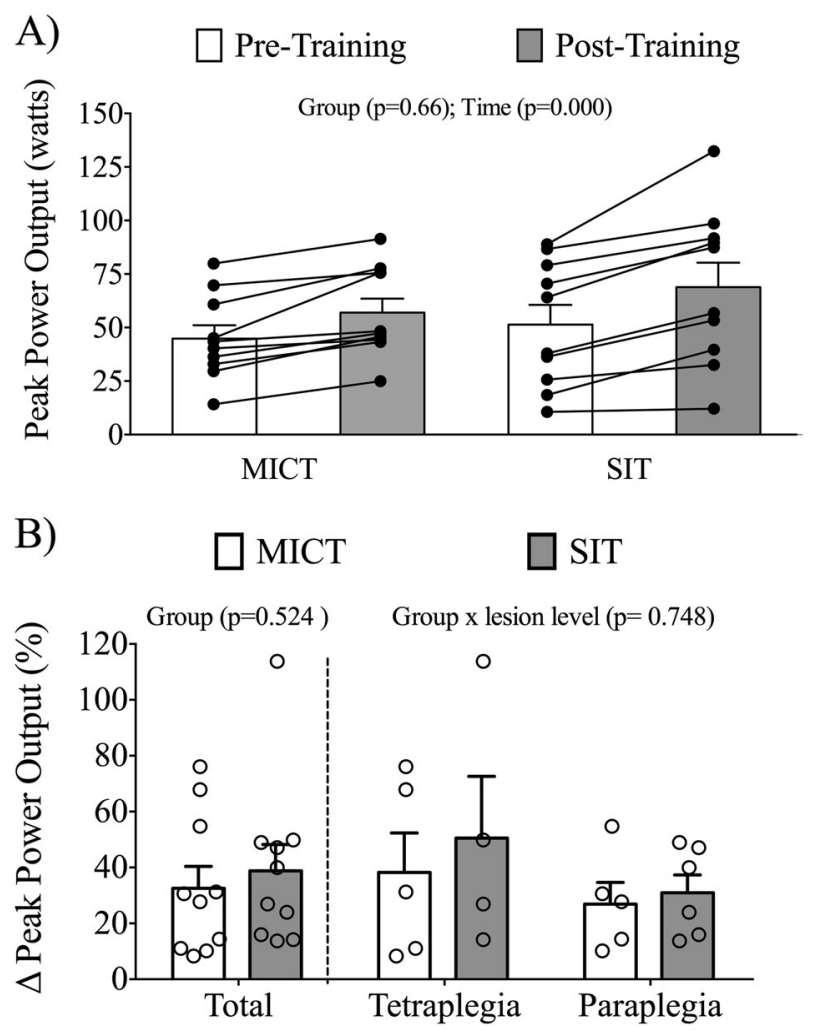

Fig. 3 Individual data (a) and relative improvements (b) in peak power output at pre-training and post-training. Mean \pm SEM are shown

heart-rate response than those performing MICT. Previously, similar values have been reported following a single session of SIT and MICT on an arm-crank ergometer in individuals with chronic SCI [19]. The heart-rate responses recorded during SIT sessions are higher than individuals with SCI undergoing over ground walking with a robotic exoskeleton [28], and performing circuit training [29], confirming the higher cardiovascular strain with SIT.

As expected, there was strong evidence of sympathetic dysfunction during training in the participants with tetraplegia, such that the heart-rate values appeared to be no different between SIT and MICT. These findings confirm that it is difficult to gauge exercise intensity in persons with tetraplegia using heart-rate values; however, a growing body of literature supports the validity of RPE scores to assess exercise intensity [30, 31]. Regardless of lesion level, we found RPE responses to be higher for individuals performing SIT $(\approx 16)$ than MICT $(\approx 12)$. An RPE of 16 corresponds to 'vigorous-intensity' exercise [30], which is an exercise intensity that individuals with sub-acute SCI undergoing inpatient rehabilitation [12], and individuals with chronic SCI living in the community [32] typically spend little to no time performing. Compared to moderateintensity exercise, engaging in vigorous-intensity exercise may attenuate cardiovascular deterioration and metabolic 
alterations following injury, ultimately reducing chronic disease risk [7, 11]. Further work is warranted to investigate the effects of performing SIT during inpatient rehabilitation
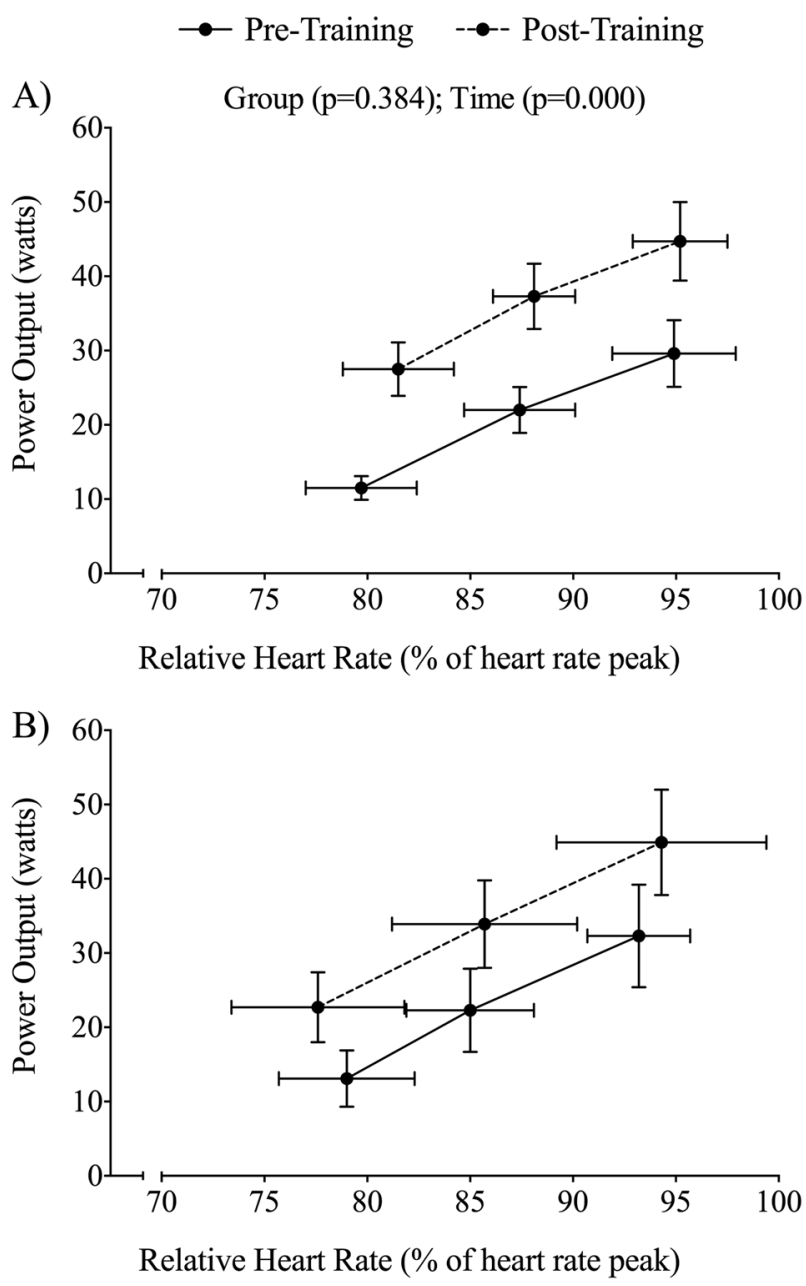

Fig. 4 Relative heart-rate/power output relationship during the submaximal arm-ergometry test at pre-training and after 5 weeks of MICT (a) or SIT (b). Mean \pm SEM are shown on altering the metabolic and cardiovascular profile of individuals with SCI.

An important finding from the current study is that there was a $39 \%$ and $33 \%$ increase in peak power output across SIT and MICT, respectively, with no difference between groups. Previous work has found comparable improvements in peak power output following MICT with arm-crank ergometry training during SCI inpatient rehabilitation, with time commitments ranging from 25 to $90 \mathrm{~min} / \mathrm{session}$ [710]. Clinically meaningful improvement standards with respect to peak power output on an arm-crank ergometer remain to be established; however, the improvements reported in the current study are considerably larger than those reported by Haisma et al., where individuals with SCI receiving traditional rehabilitation with no arm-crank ergometer training improved peak power output by only $10 \%$ [33]. In addition, we observed that individuals with tetraplegia improved peak power output to the same relative extent as individuals with paraplegia. These findings suggest that persons with upper extremity impairments can also reap the benefits of performing SIT on an arm-crank ergometer during inpatient rehabilitation.

We also observed improved power outputs during all three stages of the sub-maximal arm-crank ergometry test after both modes of training, with no differences between groups. Similar findings were reported in individuals with SCI following 9 months of twice-weekly MICT performing multi-component exercise training (aerobic exercise+resistance exercise) [6]. These improvements in sub-maximal capacity may have direct impact on the ability to perform activities of daily living. An increased power output for a given perceived effort will mean that people will be able to perform a greater amount of work before getting fatigued, which may translate into improved independence and health-related quality of life for individuals living with SCI. The fact that low-volume SIT can promote similar increases in physical capacity as MICT means that clinical

Table 3 Group responses to questionnaires

\begin{tabular}{|c|c|c|c|c|}
\hline & \multirow[t]{2}{*}{ MICT } & \multirow[t]{2}{*}{ SIT } & \multicolumn{2}{|l|}{ ANOVA } \\
\hline & & & $\Delta$ between groups & Group $p$-value \\
\hline Physical Activity Enjoyment Scale $(18-126)^{\mathrm{a}}$ & $99(16,95 \%$ CI: 87,111$)$ & $106(13,95 \%$ CI: 96, 116) & $6(15,95 \%$ CI: $-7,21)$ & 0.385 \\
\hline $\begin{array}{l}\text { Exercise Self Efficacy Scale (pre- } \\
\text { training; } 10-40 \text { ) }\end{array}$ & $36(3,95 \%$ CI: 33,38$)$ & $36(3,95 \%$ CI: 33,38$)$ & $0(3,95 \%$ CI: $-3,3)$ & 0.930 \\
\hline $\begin{array}{l}\text { Exercise Self Efficacy Scale (post- } \\
\text { training; } 10-40 \text { ) }\end{array}$ & $35(5,95 \%$ CI: 31,38$)$ & $35(5,95 \%$ CI: 31,38$)$ & $0(5,95 \%$ CI: $-5,5)$ & \\
\hline Typical pain $(3-21)^{\mathrm{a}}$ & $9(6,95 \%$ CI: 5,13$)$ & $10(4,95 \%$ CI: 6,13$)$ & $1(4,95 \%$ CI: $-4,6)$ & 0.780 \\
\hline Arm-bike specific pain $(3-21)^{a, b}$ & $7(4,95 \%$ CI: 4 to 10$)$ & 7 (3, 95\% CI: 5,9$)$ & $0(5,95 \% \mathrm{CI}:-3,3)$ & \\
\hline
\end{tabular}

Values are displayed as mean (standard deviation, 95\% CI: lower bound, upper bound). No significant differences across MICT and SIT

${ }^{\mathrm{a}}$ Administered at post-training only

${ }^{b}$ Main effect of condition (i.e., typical pain vs. arm-bike specific pain; $p=0.029$ ) 
rehabilitation specialists can now offer a new, more timeefficient, exercise training strategy to elicit improvements in their patients.

Our data demonstrate that both exercise modes were equally enjoyable and did not induce further pain. A recent scoping review concluded exercise enjoyment for SIT to be greater and/or comparable to MICT [34]. The unique nature of low-volume interval training (brief, intermittent periods of intense exercise, minimal time commitment) may lead individuals to perceive SIT to be more enjoyable than other forms of exercise [35]. Following SCI, there is an extensive reliance on the upper limbs for performing activities of daily living, which leads to a greater prevalence of shoulder overuse injuries and musculoskeletal pain in this population [36]. Given the quick generation of relatively high forces with SIT, one might predict that this type of training might pose a risk to the shoulder and/or arm musculature in people with SCI, but the results from the current study refute this, suggesting no specific risk for this type of training for individuals with sub-acute SCI undergoing inpatient rehabilitation. Further work is warranted to investigate the safety of this type of training in individuals with chronic SCI living in the community, whom, compared to individuals with sub-acute SCI, are at a greater risk of developing shoulder over-use injuries [37].

Participants reported relatively high exercise self-efficacy at pre-training, and there were no changes in self-efficacy following MICT or SIT. In contrast, a previous study found that 6 weeks of MICT on an arm-crank ergometer significantly improved exercise self-efficacy in communitydwelling individuals living with SCI [38]. Unlike community-dwelling individuals living with SCI, recently injured individuals undergoing inpatient rehabilitation are heavily monitored by physical therapists to ensure they perform exercise, and are thus not required to initiate exercise on their own. Indeed, participants in the current study reported higher pre-training exercise self-efficacy (35-36/40; Table 3) than the aforementioned study (31-33/40 [38]). This discrepancy may be attributed to the shorter time post injury and limited experience with adapting exercise to accommodate their abilities for participants in the current study [39]. Exercise self-efficacy may possibly change following discharge from inpatient rehabilitation, when persons with SCI are required to initiate exercise on their own. As improvement in exercise self-efficacy is associated with other health-related quality of life outcomes for persons with SCI [38], further work is warranted to investigate the effects of SIT in community-dwelling individuals.

\section{Study limitations}

As the study was performed in recently injured individuals in a primary rehabilitation setting, the improvements in physical capacity could be attributed to the usual rehabilitation program and natural processes of recovery following SCI. The sample size was relatively small and heterogeneous, which limited our ability to stratify based on lesion level, injury severity, or sex. For example, there were not any females randomized to perform SIT; however, previous work has not found sex differences in training adaptations to SIT [40]. It is also worth mentioning that our convenience sample did not include persons with complete tetraplegia, thus further work to investigate the effects of SIT in these individuals is warranted. The current study was also lacking body composition parameters and cardiometabolic outcomes. Finally, we did not perform any direct measurements of oxygen uptake or muscle strength, so we are unable to determine whether the improvements in physical capacity were due to changes in aerobic capacity or strength, nor whether there would be differences between the two training regimens in these outcomes.

\section{Conclusion}

This is the first study comparing SIT to MICT on an armcrank ergometer in individuals with SCI undergoing inpatient rehabilitation. Our results demonstrate that SIT improved indices of physical capacity to the same extent as MICT, despite a substantially lower exercise volume and time commitment. Both modes of exercise were equally well tolerated and highly enjoyable. Given the move towards a shortened length of stay during inpatient rehabilitation, the incorporation of SIT may represent a more viable, time-efficient alternative to MICT for improving physical capacity in this population.

\section{Data availability}

The dataset generated and/or analysed during the current study are available from the corresponding author on reasonable request

Author contributions JCM, DH, and ALH conceived and designed the study. JCM was responsible for data collection and analysis. All authors provided editorial corrections, and read and approved the final version of the manuscript.

\section{Compliance with ethical standards}

Conflict of interest The authors declare that they have no conflict of interest.

Statement of ethics We certify that all applicable institutional and governmental regulations concerning the ethical use of human volunteers were followed during the course of this research. This work was approved by the Hamilton Integrated Research Ethics Board (Project ID: 3040). 
Publisher's note: Springer Nature remains neutral with regard to jurisdictional claims in published maps and institutional affiliations.

\section{References}

1. Hachem LD, Ahuja CS, Fehlings MG. Assessment and management of acute spinal cord injury: from point of injury to rehabilitation. J Spinal Cord Med 2017;40:665-75.

2. Jacobs PL, Nash MS. Exercise recommendations for individuals with spinal cord injury. Sports Med 2004;34:727-51.

3. Bakkum AJ, Paulson TA, Bishop NC, Goosey-Tolfrey VL, Stolwijk-Swuste JM, van Kuppevelt DJ, et al. Effects of hybrid cycle and handcycle exercise on cardiovascular disease risk factors in people with spinal cord injury: a randomized controlled trial. J Rehabil Med 2015;47:523-30.

4. Bakkum AJ, de Groot S, Stolwijk-Swuste JM, van Kuppevelt DJ, Allrisc, van der Woude LH, et al. Effects of hybrid cycling versus handcycling on wheelchair-specific fitness and physical activity in people with long-term spinal cord injury: a 16-week randomized controlled trial. Spinal Cord 2015;53:395-401.

5. Nooijen CF, van den Brand IL, Ter Horst P, Wynants M, Valent LJ, Stam HJ, et al. Feasibility of handcycle training during inpatient rehabilitation in persons with spinal cord injury. Arch Phys Med Rehabil 2015;96:1654-7.

6. Hicks AL, Martin KA, Ditor DS, Latimer AE, Craven C, Bugaresti $\mathrm{J}$, et al. Long-term exercise training in persons with spinal cord injury: effects on strength, arm ergometry performance and psychological well-being. Spinal Cord 2003;41:34-43.

7. de Groot PC, Hjeltnes N, Heijboer AC, Stal W, Birkeland K. Effect of training intensity on physical capacity, lipid profile and insulin sensitivity in early rehabilitation of spinal cord injured individuals. Spinal Cord 2003;41:673-9.

8. Bizzarini E, Saccavini M, Lipanje F, Magrin P, Malisan C, Zampa A. Exercise prescription in subjects with spinal cord injuries. Arch Phys Med Rehabil 2005;86:1170-5.

9. Valent LJ, Dallmeijer AJ, Houdijk H, Slootman HJ, Janssen TW, Post MW, et al. Effects of hand cycle training on physical capacity in individuals with tetraplegia: a clinical trial. Phys Ther 2009;89:1051-60.

10. Hjeltnes N, Wallberg-Henriksson H. Improved work capacity but unchanged peak oxygen uptake during primary rehabilitation in tetraplegic patients. Spinal Cord 1998;36:691-8.

11. Ginis KA, van der Scheer JW, Latimer AE, Barrows A, Bourne C, Carruthers $\mathrm{P}$, et al. Evidence-based scientific exercise guidelines for adults with spinal cord injury: an update and a new guideline. Spinal Cord 2018;56:308-21.

12. Zbogar D, Eng JJ, Noble JW, Miller WC, Krassioukov AV, Verrier MC. Cardiovascular stress during inpatient spinal cord injury rehabilitation. Arch Phys Med Rehabil 2017;98:2449-56.

13. Craven C, Verrier M, Balioussis C, et al. Capturing Capacity in Canadian SCI Rehabilitation. Rick Hansen Institute, Vancouver, British Columbia; 2012. p 41.

14. National Spinal Cord Injury Statistical Center. 2016 Annual Statistical Report for the Spinal Cord Injury Model Systems Public Version. University of Alabama at Birmingham: Birmingham, Alabama. https://www.nscisc.uab.edu Last access: May 2018.

15. Gibala MJ, Little JP, Macdonald MJ, Hawley JA. Physiological adaptations to low-volume, high-intensity interval training in health and disease. J Physiol 2012;590:1077-84.1

16. Gillen JB, Martin BJ, MacInnis MJ, Skelly LE, Tarnopolsky MA, Gibala MJ. Twelve weeks of sprint interval training improves indices of cardiometabolic health similar to traditional endurance training despite a five-fold lower exercise volume and time commitment. PLoS ONE 2016;11:e0154075.
17. van den Akker LE, Holla JFM, Dadema T, Visser B, Valent LJ, de Groot S. Determinants of physical activity in wheelchair users with spinal cord injury or lower limb amputation: perspectives of rehabilitation professionals and wheelchair users. Disabil Rehabil 2019;1-8:12.

18. Nightingale TE, Metcalfe RS, Vollaard NB, Bilzon JL. Exercise guidelines to promote cardiometabolic health in spinal cord injured humans: time to raise the intensity? Arch Phys Med Rehabil 2017;98:1693-704.

19. Astorino TA, Thum JS. Within-session responses to high-intensity interval training in spinal cord injury. Disabil Rehabil 2018;40:444-9.

20. Harnish CR, Sabo RT, Daniels JA, Caruso D. The effects of two weeks of arm crank sprint interval training in men with chronic spinal cord injury. Int J Sports Exerc Med. 2017;3:56-59.

21. Hol AT, Eng JJ, Miller WC, Sproule S, Krassioukov AV. Reliability and validity of the six-minute arm test for the evaluation of cardiovascular fitness in people with spinal cord injury. Arch Phys Med Rehabil 2007;88:489-95.

22. Davis GM. Exercise capacity of individuals with paraplegia. Med Sci Sports Exerc 1993;25:423-32.

23. Borg GA. Psychophysical bases of perceived exertion. Med Sci Sports Exerc. 1982;14:377-81.

24. Astorino TA, Thum JS. Interval training elicits higher enjoyment versus moderate exercise in persons with spinal cord injury. $\mathrm{J}$ Spinal Cord Med. 2018;41:77-84.

25. Kendzierski D, Decarlo KJ. Physical activity enjoyment scale: two validation. Stud J Sport Ex Psych. 1991;13:50-64.

26. Kroll T, Kehn M, Ho PS, Groah S. The SCI Exercise Self-Efficacy Scale (ESES): development and psychometric properties. Int $\mathrm{J}$ Behav Nutr Phys Act 2007;4:34.

27. Pelletier CA, Ditor DS, Latimer-Cheung AE, Warburton DE, Hicks AL. Exercise equipment preferences among adults with spinal cord injury. Spinal Cord 2014;52:874-9.

28. Escalona MJ, Brosseau R, Vermette M, Comtois AS, Duclos C, Aubertin-Leheudre M, et al. Cardiorespiratory demand and rate of perceived exertion during overground walking with a robotic exoskeleton in long-term manual wheelchair users with chronic spinal cord injury: a cross-sectional study. Ann Phys Rehabil Med. 2018;61:215-23.

29. Nash MS, van de Ven I, van Elk N, Johnson BM. Effects of circuit resistance training on fitness attributes and upper-extremity pain in middle-aged men with paraplegia. Arch Phys Med Rehabil 2007;88:70-5.

30. van der Scheer JW, Hutchinson MJ, Paulson T, Martin Ginis KA, Goosey-Tolfrey VL. Reliability and validity of subjective measures of aerobic intensity in adults with spinal cord. PM R 2018;10:194-207.

31. Goosey-Tolfrey V, Lenton J, Goddard J, Oldfield V, Tolfrey K, Eston R. Regulating intensity using perceived exertion in spinal cord-injured participants. Med Sci Sport Exer 2010;42:608-13.

32. Nightingale TE, Walhin JP, Thompson D, Bilzon JL. Biomarkers of cardiometabolic health are associated with body composition characteristics but not physical activity in persons with spinal cord injury. J Spinal Cord Med 2019;42:328-37.

33. Haisma JA, Bussmann JB, Stam HJ, Sluis TA, Bergen MP, Dallmeijer AJ, et al. Changes in physical capacity during and after inpatient rehabilitation in subjects with a spinal cord injury. Arch Phys Med Rehabil 2006;87:741-8.

34. Stork MJ, Banfield LE, Gibala MJ, Martin Ginis KA. A scoping review of the psychological responses to interval exercise: is interval exercise a viable alternative to traditional exercise? Health Psychol Rev. 2017;11:324-44.

35. Jung ME, Bourne JE, Little JP. Where does HIT fit? An examination of the affective response to high-intensity intervals in comparison to continuous moderate- and continuous vigorous- 
intensity exercise in the exercise intensity-affect continuum. PLoS ONE 2014;9:e114541.

36. Cratsenberg KA, Deitrick CE, Harrington TK, Kopecky NR, Matthews BD, Ott LM, et al. Effectiveness of exercise programs for management of shoulder pain in manual wheelchair users with spinal cord injury. J Neurol Phys Ther 2015;39:197-203.

37. Ferrero G, Mijno E, Actis MV, Zampa A, Ratto N, Arpaia A, et al. Risk factors for shoulder pain in patients with spinal cord injury: a multicenter study. Musculoskelet Surg 2015;99(Suppl 1):S53-6.

38. Nightingale TE, Rouse PC, Walhin JP, Thompson D, Bilzon JLJ. Home-based exercise enhances health-related quality of life in persons with spinal cord injury: a randomized controlled trial. Arch Phys Med Rehabil 2018;99:1998-2006. e1

39. Nooijen CF, Post MW, Spooren AL, Valent LJ, Broeksteeg R, Sluis TA, et al. Exercise self-efficacy and the relation with physical behavior and physical capacity in wheelchair-dependent persons with subacute spinal cord injury. J Neuroeng Rehabil 2015;12:103.

40. Metcalfe RS, Babraj JA, Fawkner SG, Vollaard NB. Towards the minimal amount of exercise for improving metabolic health: beneficial effects of reduced-exertion high-intensity interval training. Eur J Appl Physiol. 2012;112:2767-75. 